\title{
Hip and Happening: Current Concepts in the Diagnosis and Management of Developmental Dysplasia of the Hip in 2022
}

\author{
Alaric Aroojis ${ }^{1}$ (D) $\cdot$ Rujuta Mehta ${ }^{2}$ (D)
}

C Indian Orthopaedics Association 2021

"Unlike a prosthetic arthroplasty which may need to function only for a few decades, a dislocated hip in a child needs to last a lifetime and thus its treatment should be taken with a great deal of seriousness"-quote by late Prof KT Dholakia, the pioneer of joint replacement surgery in India who began his career as a polio surgeon at the Children's Orthopaedic Hospital, Mumbai.

The hip is the most invaluable and unique joint to be studied and managed, from the womb to the tomb, by the Orthopaedic surgeon. It has held center stage of interest among researchers, whether in trauma, infection or arthroplasty, for decades. But strangely, not enough interest has been focused on the genesis of it all, that is, the Paediatric Hip. Wenger \& Bomar [1] have reviewed some historical aspects of congenital hip dislocation $(\mathrm{CDH})$, especially with respect to diagnosis and treatment through the ages. Despite being known since antiquity, with Hippocrates in 300 BC reporting a foetus with a dislocated hip joint in utero, not much attention was directed towards congenital hip dislocation in the pre-Roentgenographic era. Seldom causing more than a limp and occasional pain in adulthood, a congenital hip dislocation was often overshadowed by more life-threatening conditions such as tuberculosis and other infections of the hip joint. Most of the advances in the understanding of the etiopathogenesis, diagnosis, and treatment of $\mathrm{CDH}-$ now called developmental dysplasia of the hip (DDH), have occurred in the early \& late twentieth century. DDH is now well recognized to have a huge spectrum, ranging from

Alaric Aroojis

aaroojis@gmail.com

Rujuta Mehta

rujutabos@gmail.com

1 Department of Paediatric Orthopaedics, Bai Jerbai Wadia Hospital for Children, Acharya Donde Marg, Mumbai 400012, Maharashtra, India

2 Bai Jerbai Wadia Hospital for Children, Nanavati Max Superspecialty Hospital, Jaslok Hospital, Mumbai, India asymptomatic mild dysplasia at one end of the spectrum to a frank hip dislocation at the other end. Western literature is replete with enormous amounts of work related to $\mathrm{DDH}-$ right from basic sciences to embryology, to screening and prevention, investigative and treatment modalities, including the management of adolescent dysplasia. India, with its cultural diversity and socio-economic barriers to equitable healthcare, presents both a challenge and an opportunity to initiate unique DDH screening and preventive programmes in its paediatric population.

This 'Special Issue on DDH' was curated with a very special purpose and that was to highlight the challenges in diagnosis, the importance of screening, the role of various imaging modalities, the plethora of treatment options as the child grows, ongoing areas of research and, most importantly, to emphasize the need and implementation of a well worked out DDH care pathway. The last special issue dedicated to DDH in the Indian Journal of Orthopaedics was in 2003 under the editorship of Prof. Benjamin Joseph [2], who mentioned even then that "it is disconcerting that several of the children with hip dysplasia encountered in India are first seen after they have started walking". Rebello and Joseph [3] emphasized the problem of late presentation of DDH in India (47\% of children treated over a 3-year period were aged 1 year or older) and strongly advocated for nation-wide neonatal hip screening as a means to reduce the incidence of late-presenting DDH. 18 years later, we still do not have an infant hip screening programme that is uniformly implemented across the country. Two systematic reviews by Pandey \& Johari [4] and Harper et al. [5] in this issue analyse the effectiveness and cost analysis of various screening programmes for DDH utilized globally, while a thought-provoking editorial by Prof. Ashok Johari highlights the multipronged approach required to develop a National DDH screening programme to overcome the challenges of lack of awareness, access, knowledge, competence, resources \& legislation that are endemic to India's healthcare system [6]. Accordingly, the Paediatric Orthopaedic 
Society of India (POSI) recently partnered with seven national organizations in developing screening guidelines and a DDH care pathway that are contextual to the healthcare system in India. This was undertaken as part of a global collaboration, supported and funded by leading international organizations. The three-phased research methodology that was used to develop India-specific DDH screening guidelines are reported by Li et al. [7], in the hope that this may encourage other countries to develop similar pathways that are unique to their local healthcare systems. Mulpuri et al. [8] emphasize the importance of international, collaborative, multicenter, prospective studies that have the potential to generate high-quality evidence to guide screening and treatment practices for DDH and Pinto et al. [9] report the preliminary results of a DDH registry implemented across five Indian centres in collaboration with the International Hip Dysplasia Registry (IHDR)—-the first of it's kind in the Indian sub-continent.

Two review articles $[10,11]$ scope the incidence, diagnosis and evolution of treatment practices of DDH in India-highlighting the knowledge gaps and the lack of good-quality evidence regarding DDH management in India. Controversy still exists regarding the natural history of clinically unstable hips and radiographic dysplasia diagnosed in infancy, and Shaeffer et al. performed a systematic review to update the clinical practice guidelines published by the American Academy of Orthopaedic Surgeons (AAOS) in 2014 [12]. Traditional swaddling, which involves restrictive immobilization of the infant's lower limbs with the hips in forced extension \& adduction has been proven to be an important modifiable risk factor for DDH and Vaidya et al. [13] provide a timely review of literature regarding the need to increase awareness among Paediatricians and other healthcare professionals on the deleterious effects of swaddling on neonatal hip development and the need to encourage 'hip-safe' swaddling techniques through concerted public awareness initiatives and policy statements from professional organizations. Aarvold et al. [14] analysed the birth weights of 8874 babies born at their institution over a 24-month period and found no link between birth weight and $\mathrm{DDH}$, thus discrediting the belief that DDH is related to a packaging problem in utero. Bracing is the gold standard for the treatment of DDH in infants under 6 months of age and Merchant et al. [15] provide an excellent narrative review summarizing the current evidence regarding bracing and the various types of braces used to treat DDH.

Imaging modalities such as ultrasound and X-rays are the mainstay in the diagnosis of DDH in infancy, to supplement clinical examination. Since the 1980s, ultrasound techniques popularized by Graf \& Harcke have become the imaging tool of choice to diagnose DDH in infancy and have led to marked improvements in screening programmes throughout the world. This has, however, led to over-diagnosis and over-treatment of mild cases of hip dysplasia, and controversy still exists over universal v/s selective ultrasound screening. Furthermore, even for trained users, various DDH indices on ultrasound generally lack reproducibility, leading to errors in diagnosis. Indeed, in this issue, Omeroglu [16] reports that significant discrepancies exist in ultrasound image acquisition and assessment between examiners who had and did not have special hands-on training in the Graf technique. In an attempt to circumvent the variability in manual ultrasound image acquisition and to improve scan quality, Hareendranath et al. [17] report an innovative technique of automatically assessing hip ultrasound scan quality using artificial intelligence (AI) and deep machine learning using convolutional neural networks (CNNs), with a high degree of accuracy. As part of a quality, safety and value improvement (QSVI) initiative, Herrero et al. [18] show that point-of-care ultrasound (POCUS) performed by the clinician can significantly reduce visit time and cost of care of detecting DDH as compared to formal sonographic evaluation in the radiology suite. As a final overview of the imaging section in this special issue, Ghasseminia et al. [19] review the current status of various imaging modalities in the diagnosis \& screening for DDH, while Karnik et al. [20] provide practical guidelines on the use of ultrasound and $\mathrm{X}$-rays in the Indian context. The restrictive access to diagnostic ultrasound in India under the stringent Pre-Conception and Pre-Natal Diagnostic Techniques (PCPNDT) Act 1994 means that many parts of our country lack the availability of trained healthcare professionals who can perform and correctly interpret infant hip ultrasounds. This fact has been highlighted in the systematic review by Pandey et al. [4] and was a strong consideration in the research methodology used to develop context-specific DDH screening guidelines for India and similar countries with limited health resources [7]. In the absence of quality ultrasound, an anteroposterior (AP) radiograph of both hips may serve as a more practical screening tool in infants above the age of 4-6 months. Vogel et al.[21] report that the cumulative radiation exposure from pelvic radiographs in infants undergoing harness treatment for DDH carry a very low risk-which will go a long way in reassuring parents on the safety of repeated radiographs in the diagnosis and treatment of DDH.

Residual acetabular dysplasia (RAD) is seen in up to $30 \%$ of children treated successfully for DDH in infancy and is known to be a significant risk factor for hip osteoarthritis in early or late adulthood. Baghdadi \& Sankar [22] reviewed the literature regarding the current evidence and controversies surrounding this important topic, especially in relation to the incidence and risk factors for RAD, the diagnostic work-up, indications for treatment, and the ideal age and procedure of choice in the management of RAD. Badrinath et al. [23] quantified the 3D acetabular morphology in children with DDH compared to age- and sex-matched controls 
using pelvic CT scans and a custom 3D image processing software, and found a wide variability in acetabular coverage patterns in dysplastic hips, while Patwardhan et al. [24] evaluated acetabular development in late-diagnosed DDH treated by open reduction at a mean age of 2 years with or without a concomitant femoral osteotomy. Tolk et al. analysed the development of leg length discrepancy (LLD) and its influence on persistent acetabular dysplasia in children with DDH [25]. They observed a trend towards impaired acetabular improvement in patients with more initial LLD, emphasizing the need for thorough assessment of leg lengths early in the follow-up period and at set intervals until skeletal maturity, as per the Stanmore DDH surveillance protocol.

Until a nation-wide hip screening programme is adopted and implemented throughout the country, late-presenting DDH detected after the walking age will continue to be a challenge in India. This was highlighted by Prof. Joseph in his study 18 years ago [3], by several other studies from India over the past two decades $[10,11]$, and more recently by the IHDR-India group where the median age at presentation was over 2 years of age [9]. Lucchesi et al. [26] performed a systematic review of literature to assess the long-term outcomes of late-detected DDH treated after the walking age. It is sobering to note that after 40 years of follow-up, more than one-third of patients treated after the walking age required a total hip replacement. The treatment of DDH, especially at an older age, is fraught with complications such as re-dislocations, osteonecrosis and proximal femur growth disturbances, and residual acetabular dysplasia requiring additional surgery. Badrinath et al. [27] provide a narrative review of literature looking into the incidence and management of these important complications.

To round up this special issue on DDH we have five video submissions on important practical aspects of DDH-how to perform clinical tests for DDH [28], utility of point-of-care ultrasound in clinic [29], technical steps and interpretation of arthrograms in DDH [30], technique of closed reduction and hip spica application [31], and surgical steps of performing a medial approach open reduction [32]. Finally in this age of social media, Kodali et al. [33] performed an analysis of the frequency and content of information about DDH on Twitter and concluded that social media platforms have a great potential to promote awareness and disseminate information on DDH.

It has been a privilege and honour to helm this special issue on DDH and we are grateful to the Editor and production team of the journal for supporting us wholeheartedly in this endeavour. We thank our panel of reviewers who have shared their time and expertise in providing valuable suggestions and high-quality feedback to the authors in a timely and expedited manner, to ensure a quick turnaround between manuscript submission and eventual publication. We hope that the collection of high-quality review articles, original research and practical video techniques in this special issue will make it a 'collector's item' that will be referenced for many years to come.

Funding This article has no funding support.

\section{Declarations}

Conflict of interest The authors declare that they have no conflicts of interest.

Ethical standard This article does not contain any studies with human participants or animals performed by any of the authors.

Informed consent For this type of study formal consent is not required.

\section{References}

1. Wenger, D. R., \& Bomar, J. D. (2021). Historical aspects of DDH. JOIO. https://doi.org/10.1007/s43465-021-00470-x

2. Joseph, B. (2003). Developmental dysplasia of the hip: editorial. Ind J Orthop, 37(4), 209.

3. Rebello, G., \& Joseph, B. (2003). Late presentation of developmental dysplasia of the hip in children from southwest India-will screening help? Ind J Orthop, 34(4), 210-214.

4. Pandey, R.A., \& Johari, A. N. (2021). Screening of newborns and infants for developmental dysplasia of the hip: systematic review. JOIO. https://doi.org/10.1007/s43465-021-00409-2

5. Harper, P., Gangadharan, R., Poku, D., \& Aarvold, A. (2021). Cost analysis of screening programmes for developmental dysplasia of the hip - a systematic review. JOIO. https://doi.org/10.1007/ s43465-021-00501-7

6. Johari, A.N. (2021). Why the time is ripe for India to develop a National DDH screening programme. JOIO. https://doi.org/10. 1007/s43465-021-00573-5

7. Li, J., Aroojis, A., Mulpuri, K., Shea, K. G., \& Schaeffer, E. K. (2021). Development of a DDH care pathway for Indiaa study methodology to guide similar efforts in other countries and for other conditions. JOIO. https://doi.org/10.1007/ s43465-021-00534-y

8. Mulpuri, K., Schaeffer, E. K., \& Price, C. T. (2021). Global collaborations in developmental dysplasia of the hip. JOIO. https:// doi.org/10.1007/s43465-021-00504-4

9. Pinto, D., Aroojis, A., Shah, H., Patwardhan, S., Venkatadass, K., Sahu, C., Schaeffer, E., \& Mulpuri, K. (2021). Demographic and practice variability amongst Indian centres in a multicentre prospective observational study on developmental dysplasia of the hip. JOIO. https://doi.org/10.1007/s43465-021-00516-0

10. Chand, S., Aroojis, A., Pandey, R. A., \& Johari, A. N. (2021). The incidence, diagnosis, and treatment practices of developmental dysplasia of hip (DDH) in India: a scoping systematic review. JOIO. https://doi.org/10.1007/s43465-021-00526-y

11. Johari, A. N., Pandey, R. A., Chand, S., \& Aroojis, A. (2021). The evolution of DDH management in India. JOIO. https://doi.org/10. 1007/s43465-021-00537-9

12. Zomar, B. O., Mulpuri, K., \& Schaeffer, E. K. (2021). Examining the short-term natural history of developmental dysplasia of the hip in infancy: a systematic review. JOIO. https://doi.org/10.1007/ s43465-021-00510-6 
13. Vaidya, S., Aroojis, A., \& Mehta, R. (2021). Developmental dysplasia of hip and post-natal positioning: role of swaddling and baby-wearing. JOIO. https://doi.org/10.1007/s43465-021-00513-3

14. Hanratty, C., Thyagarajan, B., Clarke, N. M., \& Aarvold, A. (2021). There is no link between birth weight and developmental dysplasia of the hip. JOIO. https://doi.org/10.1007/ s43465-021-00465-8

15. Merchant, R., Singh, A., Dala-Ali, B., Sanghrajka, A. P., \& Eastwood, D. M. (2021). Principles of bracing in the early management of developmental dysplasia of the hip. JOIO. https://doi.org/ $10.1007 / \mathrm{s} 43465-021-00525-\mathrm{z}$

16. Ömeroğlu, H. (2021). Significant assessment discrepancies occur between the examiners having and not having further special hands-on course trainings in infantile hip ultrasonography by the Graf method. JOIO. https://doi.org/10.1007/s43465-021-00514-2

17. Hareendranath, A. R., Chahal, B. S., Zonoobi, D., Sukhdeep, D., \& Jaremko, J. L. (2021). Artificial intelligence to automatically assess scan quality in hip ultrasound. JOIO. https://doi.org/10. 1007/s43465-021-00455-w

18. Herrero, C., Colon, Y., Nagapurkar, A., \& Castañeda, P. (2021). Point-of-care ultrasound reduces visit time and cost of care for infants with developmental dysplasia of the hip. JOIO. https:// doi.org/10.1007/s43465-021-00541-z

19. Ghasseminia, S., Hareendranath, A. R., \& Jaremko, J. L. (2021). Narrative review on the role of imaging in DDH. JOIO. https:// doi.org/10.1007/s43465-021-00511-5

20. Karnik, A., Lawande, A., Lawande, M. A., Patkar, D., Aroojis, A., \& Bhatnagar, N. (2021). Practice essentials of imaging in early diagnosis of DDH. JOIO. https://doi.org/10.1007/ s43465-021-00539-7

21. Vogel, E., Leaver, T., Wall, F., Johnson, B., Uglow, M., \& Aarvold, A. (2021). Repeated pelvic radiographs in infants, after harness treatment for developmental dysplasia of the hip, carry very low radiation risk. JOIO. https://doi.org/10.1007/s43465-021-00438-x

22. Baghdadi, S., \& Sankar, W. N. (2021). Residual acetabular dysplasia in the reduced hip. JOIO. https://doi.org/10.1007/ s43465-021-00515-1

23. Badrinath, R., Jeffords, M. E., Bomar, J. D., Ahmed, S. I., Pennock, A. T., \& Upasani, V. V. (2021). 3D characterization of acetabular deficiency in children with developmental dysplasia of the hip. JOIO. https://doi.org/10.1007/s43465-021-00458-7

24. Patwardhan, S., Madegowda, A., \& Sancheti, P. (2021). Evaluation of acetabular development following open reduction of developmental dysplasia of hip in children after walking age. JOIO. https://doi.org/10.1007/s43465-021-00527-x

25. Tolk, J. J., Merchant, R., Eastwood, D. M., Buddhev, P., \& Hashemi-Nejad, A. (2021). The development of leg length difference and influence on persistent dysplasia in patients with developmental dysplasia of the hip. JOIO. https://doi.org/10.1007/ s43465-021-00492-5

26. Lucchesi, G., Sacco, R., Zhou, W., Li, Y. Q., Li, L., \& Canavese, F. (2021). DDH in the walking age: Review of patients with long-term follow-up. JOIO. https://doi.org/10.1007/ s43465-021-00531-1

27. Badrinath, R., Orner, C., Bomar, J. D., \& Upasani, V. V. (2021). Narrative review of complications following DDH treatment. JOIO. https://doi.org/10.1007/s43465-021-00550-y

28. Bhaskar, A., Doshi, C., Nagda, T., \& Bhaskar, D. (2021). Video technique on clinical tests for DDH: newborn to walking age. JOIO. https://doi.org/10.1007/s43465-021-00528-w

29. Herrero, C., Jejurikar, N., Kanakalamedala, A., Iban, Y. C., \& Castañeda, P. (2021). Point of care ultrasound as performed by the clinician for the enhancement of physical examination: a technique guide for developmental hip dysplasia. JOIO. https://doi. org/10.1007/s43465-021-00570-8

30. Agashe, M. V., Kapoor, D., \& Vaidya, S. V. (2021). Technique and interpretation of arthrogram for developmental dysplasia of the hip. JOIO. https://doi.org/10.1007/s43465-021-00493-4

31. Nagda, T., \& Dhamele, J. (2021). Closed reduction, arthrogram and spica application for DDH. JOIO. https://doi.org/10.1007/ s43465-021-00542-y

32. Naik, P. (2021). Video technique on medial open reduction for DDH. JOIO. https://doi.org/10.1007/s43465-021-00502-6

33. Kodali, N., Sidhu, B. V., So, J., Habib, E., Robillard, J. M., Mulpuri, K., \& Schaeffer, E. K. (2021). Content analysis of frequency of information about developmental dysplasia of the hip on Twitter. JOIO. https://doi.org/10.1007/s43465-021-00553-9

Publisher's Note Springer Nature remains neutral with regard to jurisdictional claims in published maps and institutional affiliations. 